\title{
Hemodiálisis sin heparina. Sistema de lavado continuo doble: ¿mejora el sistema de lavado continuo prefiltro?
}

\author{
Francisco Belchí Rosique \\ Antonia Alfaro Cuenca
}

Hospital Santa María del Rosell

Cartagena

\section{RESUMEN}

Cuando existe contraindicación para la anticoagulación, se deben utilizar métodos alternativos. Ante problemas de coagulación del circuito durante la sesión de hemodiálisis un sistema de "lavado continuo" del equipo puede ser una alternativa válida. El resultado de la técnica puede mejorarse aumentando la dilución de la sangre en la cámara venosa.

Denominamos lavado continuo doble a la técnica empleada. Consiste en la administración continua y simultanea durante la sesión de hemodiálisis de suero salino fisiológico por la cámara arterial y, por la cámara venosa, administramos suero fisiológico alternándolo con bicarbonato $1 / 6 \mathrm{M}$, para evitar la posible acidosis provocada por los elevados volúmenes de suero salino, a una velocidad de 1 litro/h por la cámara arterial y de $500 \mathrm{ml} / \mathrm{h}$ por la cámara venosa, utilizando para ello dos bombas de perfusión IVAC con los sistemas conectados directamente a dichas cámaras. Se realizaron 50 sesiones con lavado simple (L1) y 50 con lavado continuo doble (L2). Se observó la aparición de coágulos en las cámaras arterial y venosa, el cambio de líneas y la necesidad de finalizar prematuramente la sesión por coagulación del circuito.

Con L2 la cámara arterial permaneció limpia en el $80 \%$ de los casos, frente al $50 \%$ con $\mathrm{L} 1$; en la cámara venosa aparecen coágulos importantes en 29 sesiones y se coagula en

\section{Correspondencia: \\ Francisco Belchí Rosique \\ Servicio de Nefrología \\ Hospital Santa María del Rosell \\ Paseo Alfonso XIII, 61 \\ 30203. Cartagena}

Correo electrónico: franciscobelchi@telefonica.net
8 sesiones con $L 1$, mientras que con $L 2$ se coagula en 4 ocasiones. Con $\mathrm{L} 1$ se finalizó prematuramente el tratamiento en 5 sesiones, mientras con L2 se finalizó en 2 . La coagulación del $50 \%$ de las fibras al final de la sesión se observó en el $60 \%$ de los casos con L1, mientras que con L2 se observo en el $34 \%$.

El lavado continuo doble es una técnica de enfermería eficaz cuando existen contraindicaciones para una anticoagulación convencional.

\section{PALABRAS CLAVE: HEMODIALISIS SIN HEPARINA LAVADO CONTINUO PREFILTRO LAVADO CONTINUO DOBLE}

\section{HEPARIN-FREE HAEMODIALYSIS. DOUBLE CON- TINUOUS RINSING SYSTEM: IS IT BETTER THAN THE CONTINUOUS PREFILTER RINSING SYS- TEM?}

\begin{abstract}
When anti-coagulation is contraindicated, alternative methods have to be used. When faced with circuit coagulation problems during the haemodialysis session a "continuous rinsing" system of the equipment may be a valid alternative. The result of the technique can be improved by increasing the dilution of the blood in the vein chamber.

Double continuous rinsing is the name given to the technique used. It consists of the continuous and simultaneous administration during the haemodialysis session of physiological saline solution through the arterial chamber, while in the vein chamber we administer physiological saline solution alternated with bicarbonate $1 / 6 \mathrm{M}$, to avoid possible acidosis
\end{abstract}


caused by the high volumes of saline solution, at a rate of 1 litre/ $h$ through the arterial chamber and $500 \mathrm{ml} / \mathrm{h}$ through the vein chamber, using two IVAC infusion pumps with the systems connected directly to these chambers. 50 sessions were carried out with simple rinsing (R1) and 50 with double continuous rinsing (R2). The appearance of clots in the arterial and vein chambers, the change of lines and the need to end the session early due to coagulation of the circuit were all observed.

With $\mathrm{L} 2$ the arterial chamber remained clean in $80 \%$ of cases, compared to $50 \%$ with $\mathrm{L} 1$; in the vein chamber, significant clots appeared in 29 sessions and coagulation occurred in 8 sessions with $L 1$, while with $L 2$ coagulation occurred on 4 occasions. With L1 the treatment had to be ended early in 5 sessions, while with L2 this occurred in 2 . Coagulation of $50 \%$ of the fibres at the end of the session was observed in $60 \%$ of cases with $L 1$, while with $L 2$ this was observed in $34 \%$.

Double continuous rinsing is an effective nursing technique when there are contraindications to conventional anti-coagulation.

KEYWORDS: HEPARIN-FREE HAEMODIALYSIS CONTINUOUS PREFILTER RINSING

DOUBLE CONTINUOUS RINSING

\section{INTRODUCCIÓN}

La utilización de heparina sigue siendo el sistema de anticoagulación estándar de mayor aplicación, a pesar del riesgo de hemorragia que conlleva ${ }^{1}$. En pacientes con riesgo de sangrado, la utilización de dosis bajas de heparina ha supuesto una ventaja en la reducción del riesgo de hemorragia y coagulación del dializador. En pacientes que presentan hemorragia activa 0 en los que existe contraindicación para la anticoagulación, se hace obligada la abstención de heparina, debiendo utilizar métodos alternativos. Tradicionalmente se ha demostrado eficaz la administración de bolos de suero fisiológico de manera intermitente durante la sesión de hemodiálisis².

Un sistema de lavado continuo para la diálisis sin heparina es superior a la administración de bolos de suero fisiológico de forma discontinua para evitar la coagulación del circuito sanguíneo en hemodiálisis ${ }^{3}$. La técnica consiste en la administración prefiltro, en la línea arterial, de suero fisiológico a una velocidad constante de 1 litro/hora, produciendo un efecto de dilución desde el inicio del circuito sanguíneo.

Tras incorporar esta técnica en nuestra unidad, coincidimos con otros autores en su eficacia, y en otras ventajas como: sencillez de aplicación, eliminación del riesgo de retrofiltra- ción, reducción de errores por su automatización y disminución de la carga de trabajo del personal de enfermería.

Sin embargo, del mismo modo que observamos buenos resultados en la coagulabilidad de la línea arterial y el dializador, no ocurre lo mismo con la cámara venosa, donde observamos una frecuente aparición de coágulos que nos obligan, en ocasiones, a desconectar antes de tiempo al paciente, cambiar la línea venosa 0 , en el peor de los casos, desechar todo el sistema ante la imposibilidad de retornar la sangre, con la consiguiente pérdida hemática que supone.

Esto es lógico, teniendo en cuenta que con la utilización de este método, la hemoconcentración es mayor a la salida del dializador, lo que unido a la estasis de la sangre en la cámara venosa, favorece la formación de coágulos.

Siguiendo este razonamiento, nos planteamos la hipótesis de que, aumentando la dilución y realizándola también a la salida del dializador, en la cámara venosa, podríamos reducir la formación de coágulos en dicha cámara, al estar haciendo una dilución directa y continua en la misma. De esta forma se pueden mejorar los resultados de la técnica, aunque sea necesario aumentar la ultrafiltración y por ende, aumenta también la hemoconcentración.

\section{OBJETIVOS}

- Describir una nueva técnica de lavado continuo para realizar hemodiálisis sin heparina.

- Comparar la eficacia del sistema de lavado continuo doble frente al sistema de lavado continuo prefiltro.

\section{PACIENTES Y MÉTODO}

Realizamos un estudio prospectivo aleatorizado con 25 pacientes, 11 hombres (44\%) y 14 mujeres (56\%), con una edad media de 60,60 años $\pm 13,05$ (27-78). El tiempo de permanencia en diálisis es de 88,60 meses $\pm 83,57$ (2-265). La distribución según la enfermedad de base es: intersticial 4 (16\%), vascular $2(8 \%)$, diabetes mellitus $6(24 \%)$, poliquística 3 $(12 \%)$, desconocida $7(28 \%)$, y otras $3(12 \%)$.

Los lavados fueron utilizados por las siguientes causas: cirugía 9 ocasiones (36\%), hemorragia digestiva $6(24 \%)$, radiología intervencionista $7(28 \%)$, y alteraciones en la coagulación 3 $(12 \%)$.

A todos los pacientes se les aplicaron ambos sistemas de lavado en sesiones de hemodiálisis convencional: 50 sesiones con lavado continuo prefiltro $(\mathrm{L} 1)$ y 50 sesiones con la nueva técnica de lavado continuo doble (L 2$)$. 


\section{Descripción de la técnica:}

La técnica de lavado continuo doble consiste en la administración continua y simultanea durante la sesión de hemodiálisis de suero salino fisiológico por la cámara arterial y, por la cámara venosa, suero fisiológico alternándolo con bicarbonato 1 / $6 \mathrm{M}$, para evitar la posible acidosis provocada por los elevados volúmenes de suero salino, a una velocidad de 1 litro/h por la cámara arterial y de $500 \mathrm{ml} / \mathrm{h}$ por la cámara venosa, utilizando para ello dos bombas de perfusión IVAC con los sistemas conectados directamente a dichas cámaras.

Así, por ejemplo, para una hemodiálisis convencional de 4 horas, necesitaríamos un total de 6 litros. Administraríamos 4 litros de suero fisiológico por la cámara arterial. Por la cámara venosa administraríamos 2 litros, de los cuales 1 litro sería de suero salino y 1 litro de bicarbonato $1 / 6 \mathrm{M}$, alternándolos en sueros de $500 \mathrm{ml}$.

Empleamos dializadores de alta permeabilidad en todas las sesiones: polisulfonas (37 sesiones), polietersulfonas (43 sesiones) y poliamidas (20 sesiones).

Registramos en cada sesión el hematocrito del paciente, acceso vascular, tiempo de diálisis, flujo sanguíneo $(\mathrm{Q} b)$, transfusión intradiálisis, presión venosa (PV), Ultrafiltración (UF), volumen de suero perfundido, estado del dializador y de las cámaras arterial y venosa según la escala:

\section{Cámaras arterial y venosa}

- 0: limpias

- 1: pequeño coágulo

- 2: coágulo importante

- 3: totalmente coagulada

\section{Estado del dializador}

- 0: limpio o capilares sueltos coagulados (más de un $80 \%$ limpio)

- 1: dializador con restos de fibrina o coagulado formando una franja delgada (70\% limpio)

- 2: dializador formando una gran franja gruesa o dos delgadas (50-60\% limpio)

- 3: coagulado más del $50 \%$

- 4: totalmente coagulado.

Análisis estadístico: El análisis estadístico lo realizamos mediante un programa SPSS 13.0 para Windows. Los estadísticos descriptivos utilizados son porcentajes y frecuencias para las variables cualitativas, y media, desviación estándar (DE) y rango para las cuantitativas. Para el contraste de las variables empleamos t-Student en el caso de las cuantitativas y Chi-cuadrado para las cualitativas. A plicamos el estadístico exacto de Fischer para tablas de contingencia $2 \times 2$. A ceptamos significación estadística para $p<0.05$.

\section{RESULTADOS}

No existen diferencias significativas entre los dializadores empleados, ni entre los accesos vasculares en ambos sistemas de lavado. En el sistema L1 se desconectó antes de tiempo por aumento de presión venosa o coagulación del sistema en un $10 \%$ de los casos (5 sesiones). En el sistema L2, en un $4 \%$ de los casos (2 sesiones).

Así mismo, tuvimos que disminuir el Q b por aumento de la PV en un $4 \%$ de los casos (2 sesiones) en $L 1$, frente a un $2 \%$ ( 1 sesión) en la L2.

Cambios de dializador o líneas. Fue necesario cambiar el dializador o las líneas debido al aumento de la presión venosa o coagulación del circuito en un $22 \%$ de los casos (11 sesiones) en $L 1$, mientras que en $L 2$ sólo ocurrió en un $8 \%$ de los casos (4 sesiones).

En cuanto a los cambios de líneas arteriales requeridos, sólo fue necesario en una ocasión en $L 1$, y ninguna en $L 2$. Cambiamos el dializador en 2 ocasiones en L 1 , y en 1 ocasión en el L2. Sin embargo, en las líneas venosas utilizadas, sí encontramos significación estadística. Obtuvimos diferencias significativas a favor del L2 en el estado de la cámara arterial y en el estado de la cámara venosa.

En cuanto al estado del dializador, si resumimos los dializadores según su estado en dos grupos: $<50 \%$ de fibras coaguladas y $>50 \%$ de fibras coaguladas, se obtienen nuevamente diferencias con significación estadística entre los dos tipos de lavados. Estos y los demás parámetros estudiados se recogen en las tablas 1 y 2 .

\begin{tabular}{lccc} 
& Lavado 1 & Lavado 2 & p \\
\hline To diálisis programado (minutos) & $234,90 \pm 16,49$ & $234,60 \pm 16,81$ & ns \\
\hline To diálisis real (minutos) & $233,20 \pm 16,89$ & $233,30 \pm 17,25$ & ns \\
\hline Qb medio (mil/min) & $327,85 \pm 53,62$ & $317,90 \pm 56,42$ & ns \\
\hline PV media (mmHg) & $163,17 \pm 33,51$ & $162,77 \pm 34,85$ & ns \\
\hline PV máxima (mmHg) & $196,94 \pm 59,63$ & $189,70 \pm 52,55$ & ns \\
\hline UF programada (gr) & $2376 \pm 883,54$ & $2238 \pm 767,15$ & ns \\
\hline UF real (gr) & $2518 \pm 890,70$ & $2330 \pm 728,50$ & ns \\
\hline Hb (gr/dl) & $11,25 \pm 1,11$ & $11,24 \pm 1,19$ & ns \\
\hline Plaquetas & $234120 \pm 90542$ & $252140 \pm 95591$ & ns \\
\hline To protrombina & $1,39 \pm 0,35$ & $1,38 \pm 0,34$ & ns \\
\hline Hipotensiones (sesiones) & 8 & 4 & ns
\end{tabular}

Tabla 1. Características de las hemodiálisis. 


\section{DISCUSIÓN}

De los resultados obtenidos podemos decir que el sistema de lavado continuo doble mejora significativamente el estado de las líneas y del dializador. Cabe destacar que la cámara arterial permanece limpia en el $80 \%$ de los casos (40 sesiones) del lavado 2 , mientras que en el lavado 1 , el $50 \%$ de los casos $(25$ sesiones) presentan restos de fibrina en mayor o menor medida.

\begin{tabular}{|c|c|c|c|}
\hline & LAVADO 1 & LAVADO 2 & $p$ \\
\hline $\begin{array}{l}\text { Desconexión antes de } \\
\text { tiempo (>PV) (sesiones) }\end{array}$ & 5 & 2 & \\
\hline Disminución del Qb (>PV) (sesiones) & 2 & 1 & \\
\hline Cambios de dializador o líneas: & 11 & 4 & 0.045 \\
\hline Línea arterial & 1 & 0 & \\
\hline Dializador & 2 & 1 & \\
\hline Línea venosa & 11 & 4 & \\
\hline \multicolumn{4}{|l|}{ Estado Cámara Arterial: } \\
\hline 0 & 25 & 40 & 0.007 \\
\hline 1 & 21 & 8 & \\
\hline 2 & 4 & 2 & \\
\hline 3 & 0 & 0 & \\
\hline \multicolumn{4}{|l|}{ Estado Cámara Venosa: } \\
\hline 0 & 5 & 18 & $p<0.001$ \\
\hline 1 & 8 & 18 & \\
\hline 2 & 29 & 12 & \\
\hline 3 & 8 & 2 & \\
\hline \multicolumn{4}{|l|}{ Estado del Dializador: } \\
\hline 0 & 8 & 19 & 0.039 \\
\hline 1 & 12 & 14 & \\
\hline 2 & 16 & 10 & \\
\hline 3 & 14 & 7 & \\
\hline 4 & 0 & 0 & \\
\hline
\end{tabular}

Tabla 2. Comparativa entre ambos tipos de lavado.

En cuanto al estado de la cámara venosa, en el lavado continuo prefiltro encontramos que en el $74 \%$ de las ocasiones (37 sesiones), ésta queda con coágulo importante (29 sesiones) 0 coagulada (8 sesiones), lo que nos obliga a cambiar la línea venosa en 11 ocasiones, mientras que esto sólo ocurre en 4 oca- siones con el lavado continuo doble ( $p$ 0.045), o bien a desconectar antes de tiempo al paciente en 5 ocasiones. En el lavado continuo doble sólo se desconectó al paciente antes de tiempo en 2 ocasiones.

Respecto al dializador, no hay ningún caso de filtro totalmente coagulado en ambos tipos de lavado. En el lavado continuo prefiltro encontramos que en el $60 \%$ de los casos, más del $50 \%$ de las fibras de los dializadores están coaguladas, reduciéndose la superficie efectiva del dializador ${ }^{4}$. Sin embargo, en el lavado continuo doble esto ocurre sólo en un $34 \%$ de los casos ( $p$ 0.008). No encontramos diferencias significativas en el resto de variables estudiadas.

\section{CONCLUSIÓN}

Podemos concluir nuestro estudio diciendo que el lavado continuo doble no soluciona el problema de coagulación de la cámara venosa, pero sí lo mejora de manera significativa con respecto al lavado continuo prefiltro. Del mismo modo, mejora significativamente el estado del dializador al finalizar la sesión de diálisis, y el estado de la cámara arterial.

Por lo tanto, se puede afirmar que el lavado continuo doble es una técnica de enfermería eficaz para realizar hemodiálisis sin heparina y que mejora el lavado continuo prefiltro.

\section{BIBLIOGRAFÍA}

1. Piera L, Fort J . Anticoagulación. En: Valderrábano F. Tratado de hemodiálisis. Barcelona: Editorial Médica Jims; 1999: 117-123.

2. Sanders PW, Taylor H, Curtis J J . H emodialysis without anticoagulation. Am J Kidney Dis. 1985; 5: 32-35.

3. Sánchez Villar I, Lorenzo González J L, García Ruiz A, Cabello $\mathrm{O}, \mathrm{A}$ bad $\mathrm{C}$. Valido $\mathrm{P}$ et al. Una nueva forma de dializar sin heparina: sistema de lavado continuo. Libro de Comunicaciones presentadas al XXV Congreso Nacional de la SEDEN. O viedo. 2000: 18-22.

4. http:// www.renal.org.ar Adecuación de diálisis: Resumen de las normas Doqy. Lavorato C. 2000; 12. 